


\title{
Aprestamiento: saberes y prácticas de una experiencia en educación musical para la primera infancia ${ }^{1}$
}

\section{Resumen}

En este artículo se muestra el resultado de la sistematización de la experiencia en educación musical para la primera infancia, denominada aprestamiento, desarrollada en el Taller de Música². Consiste en una propuesta surgida desde la pedagogía musical, estructurada en 6 componentes de clases que organizan y estructuran los contenidos, para favorecer aspectos del desarrollo de niños ${ }^{3}$ entre 2 y 5 años, época en la cual el estímulo recibido contribuye a facilitar los procesos de iniciación a la lectoescritura musical y la práctica instrumental. La experiencia realizada por la maestra María Teresa Martínez, reconocida en el medio pedagógico musical como "Pitti Martinez", refleja la comprensión adquirida a lo largo de los años sobre la importancia de la música en los procesos de desarrollo físico, emocional, cognitivo y social de los niños, con el fin de aportar a su presente y a su futuro, y de esta forma contribuir en alguna medida a una sociedad más amable.

Palabras clave: educación musical, primera infancia, aprestamiento, Pitti Martínez Taller de Música, Pittigrafias.

\section{Readiness: Knowledge and Practices of an Experience in Musical Education for Early Childhood}

\begin{abstract}
This article presents the results of the systematization of an experience in music education for toddlers and little children, herein named "Readiness", which was carried on in the Taller de Música. Is consists of a proposal emerged from the musical pedagogy, where the contents are organized and configured in six components so structured as to stimulate developmental aspects of children aged 2 to 5 . The experience held by María Teresa Martínez, teacher in music education and better known in the pedagogical environment as "Pitti Martinez", resumes the comprehension acquired during all these years regarding the importance of music in the processes of physical, emotional, cognitive and social development of children, and aims at providing for their present and future, thus helping to some extent to build a gentler society.

Keywords: Music Education, Early Childhood, Readiness, Pitti Martínez Taller de Música, Pittigrafías.

\section{Aprestamento: saberes musicais e práticas de uma experiência em educação musical para a primeira infância}

\section{Resumo}

Neste artigo mostram-se os resultados da sistematização da experiência em educação musical para a primeira infância, denominada "Aprestamento", desenvolvida no Taller de Música ${ }^{4}$. Consiste numa proposta surgida desde a pedagogia musical, estruturada em seis componentes de aulas que organizam e estruturam os conteúdos, para favorecer aspectos do desenvolvimento de crianças entre dois e cinco anos de idade. A experiência realizada pela professora Maria Teresa Martínez (reconhecida no médio pedagógico musical como "Pitti Martínez"), reflete a compreensão adquirida ao longo dos anos sobre a importância da música nos processos de desenvolvimento físico, emocional, cognitivo e social das crianças, com o fim de aportar a sua contemporaneidade e a seu futuro, e desta forma contribuir em alguma medida a uma sociedade mais amável.

Palavras chave: educação musical, primeira infância, aprestamento, Pitti Martínez, Oficina de Música, Pittigrafias.

\footnotetext{
1 Esta sistematización se realizó como trabajo de grado para optar por el título de Magíster en Desarrollo Educativo y Social, programa de la Fundación Cinde en Convenio con la Universidad Pedagógica Nacional, adelantado por Pitti Martínez y Diana programa de la Fundación Cinde en Convenio con la Universidad Pedagógica Nacional, adelantado por Pitti Martion de esa institución para la Cohorte UPN-37, bajo la dirección de la maestra Marina Camargo Abello 2 El Taller de Música está ubicado en Bogotá, es una propuesta individual en la que se brinda educación no formal en música a niños de dos años en adelante.

3 En una perspectiva de género, debe entenderse para efectos de este documento que también están incluidas las niñas.

4 O Taller de Música está situado em Bogotá, é uma proposta individual, na que se oferece educação não formal em música para crianças de dois anos em diante.
} 


\section{Introducción}

La educación musical contemporánea se plantea como un proceso destinado fundamentalmente al desarrollo de las facultades humanas, es decir, no está en función única y exclusiva de los músicos, sino que está dirigida hacia una formación integral que potencie las cualidades intrínsecamente humanas y que ayude a la formación de seres sensibles, inteligentes y hábiles para la convivencia.

En tal sentido, cobra importancia la sistematización de experiencias $^{5}$ como ejercicio investigativo de prácticas educativas, para alcanzar comprensiones y abrir posibilidades de compartir saberes pedagógicos (Torres, 1998). En el presente caso, bajo el título de Saberes y prácticas de una experiencia musical para la primera infancia en la ciudad de Bogotá, se realizó una sistematización con el objetivo de develar los fines y las intencionalidades pedagógicas del aprestamiento.

Durante el proceso investigativo se hizo una reconstrucción histórica, se identificaron las prácticas que estructuran el aprestamiento y se exploraron los aprendizajes de los cuales emergieron nuevos saberes y una comprensión más amplia sobre el hacer de la maestra.

Para dar mayor claridad, es conveniente recurrir al significado ${ }^{6}$ de aprestamiento desde las palabras que lo componen, el cual coincide con su sentido: aprestar, que implica preparar, disponer lo necesario para algo; y el sustantivo miento, que significa acción y efecto. Por tanto, en el contexto de esta investigación, se entenderá al aprestamiento ${ }^{7}$ como "estar listo para la vida”. Tiene su origen en una iniciativa individual surgida hace aproximadamente veinte años, la cual ha estado en permanente construcción. Se fundamenta en metodologías de educación musical y en aproximaciones de otras disciplinas, como la psicología cognitiva y las neurociencias. Su evolución es producto de un proceso de exploración, revisión continua de las prácticas, búsqueda, selección, creación de materiales y repertorios.

$\mathrm{Al}$ investigar se encontró que la experiencia refleja aspectos personales de la maestra, como su constante interés por encontrar nuevos saberes que sean aportantes a sus prácticas, como el hecho de que la música haya sido una decisión y un motivo para ser y hacer, la importancia de generar en los niños recuerdos para atesorar y sobre todo, fomentar la alegría de la vida. Dichos aspectos muestran que el aprestamiento, además de aportar a la educación inicial, es una invitación a los maestros a ser más conscientes de su potencialidad y del poder transformador de sus prácticas.

\footnotetext{
5 La sistematización de experiencias da lugar a nuevos aprendizajes y permite a los actores reorientar o consolidar acciones que potencialicen sus prácticas, les suscita nuevos interrogantes e incita a una toma de conciencia y reconocimiento de su saber. "A toda sistematización le antecede una práctica. A diferencia de otros procesos investigativos a éste le antecede un 'hacer' que puede ser recuperado, recontextualizado, textualizado, analizado y reinformado a partir del conocimiento adquirido a lo largo del proceso" (Ghiso, 1999, p. 9). 6 Este significado fue tomado del diccionario de la Real Academia Española de la Lengua. Recuperado de http://dle.rae.es/?id=3IppS5w

7 El Aprestamiento tal como se ha desarrollado surgió como parte de la preparación necesaria para un desempeño instrumental y la lectoescritura requerida en el mismo, por parte de los alumnos del Taller de música de la maestra Pitti Martínez. Se dice que por parte de los alumnos del Taller de música de la maestra Pitti Martínez. Se dice que
es "estar listo para la vida" por los beneficios adicionales que aporta el trabajo musical sistemático a los procesos de atención sostenida en el presente, pensamiento asociativo y su consecuente optimización de la memoria, incluyendo en esta, las memorias melódica, rítmica, lingüistica, tímbrica, formal, armónica, kinestésica, métrica, digital, nominal, que contribuyen a engrosar el cuerpo calloso en el cerebro (Mannes, 2009). En consecuencia el término empleado en la experiencia como Aprestamiento tiene una connotación diferente al usado en la Política Pública.
}

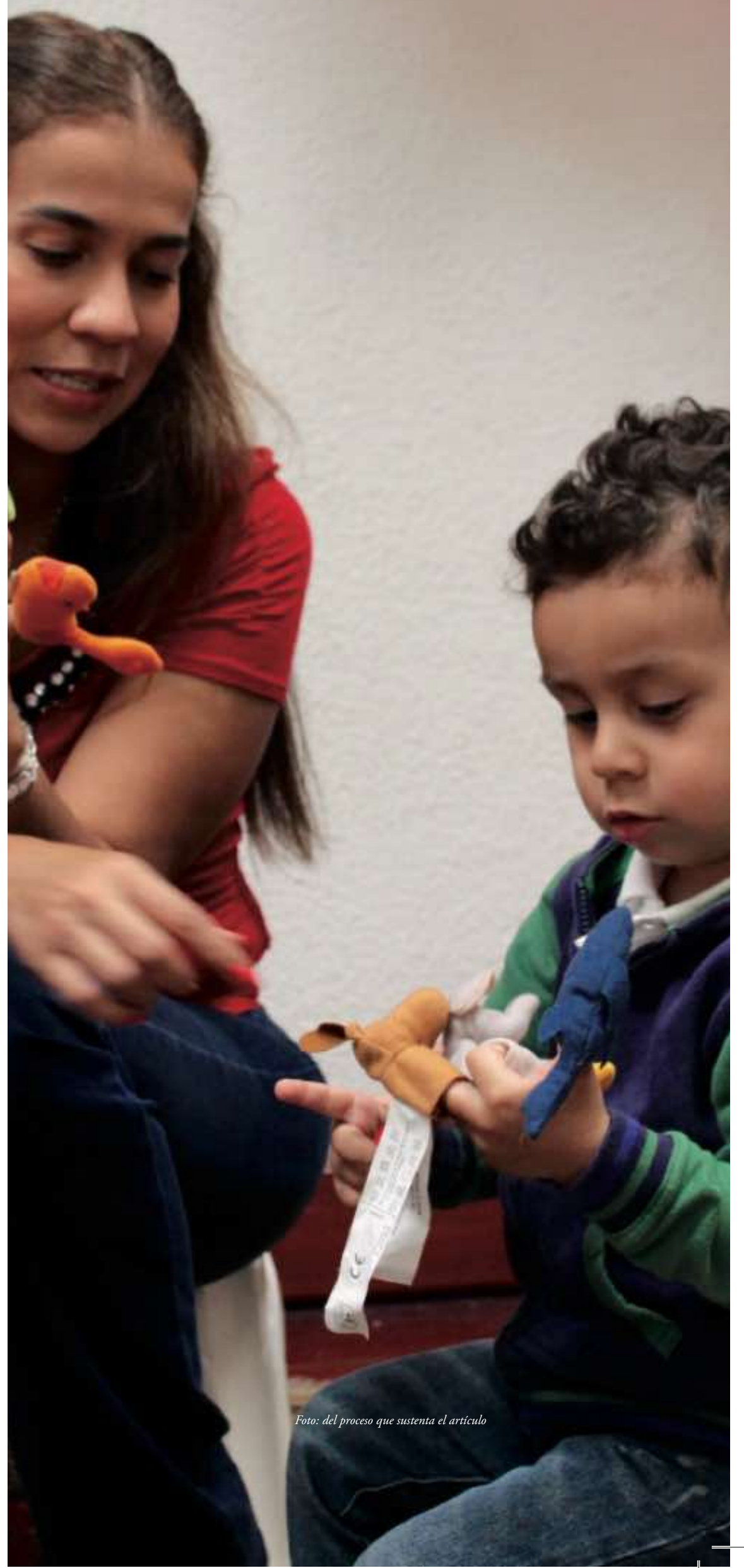




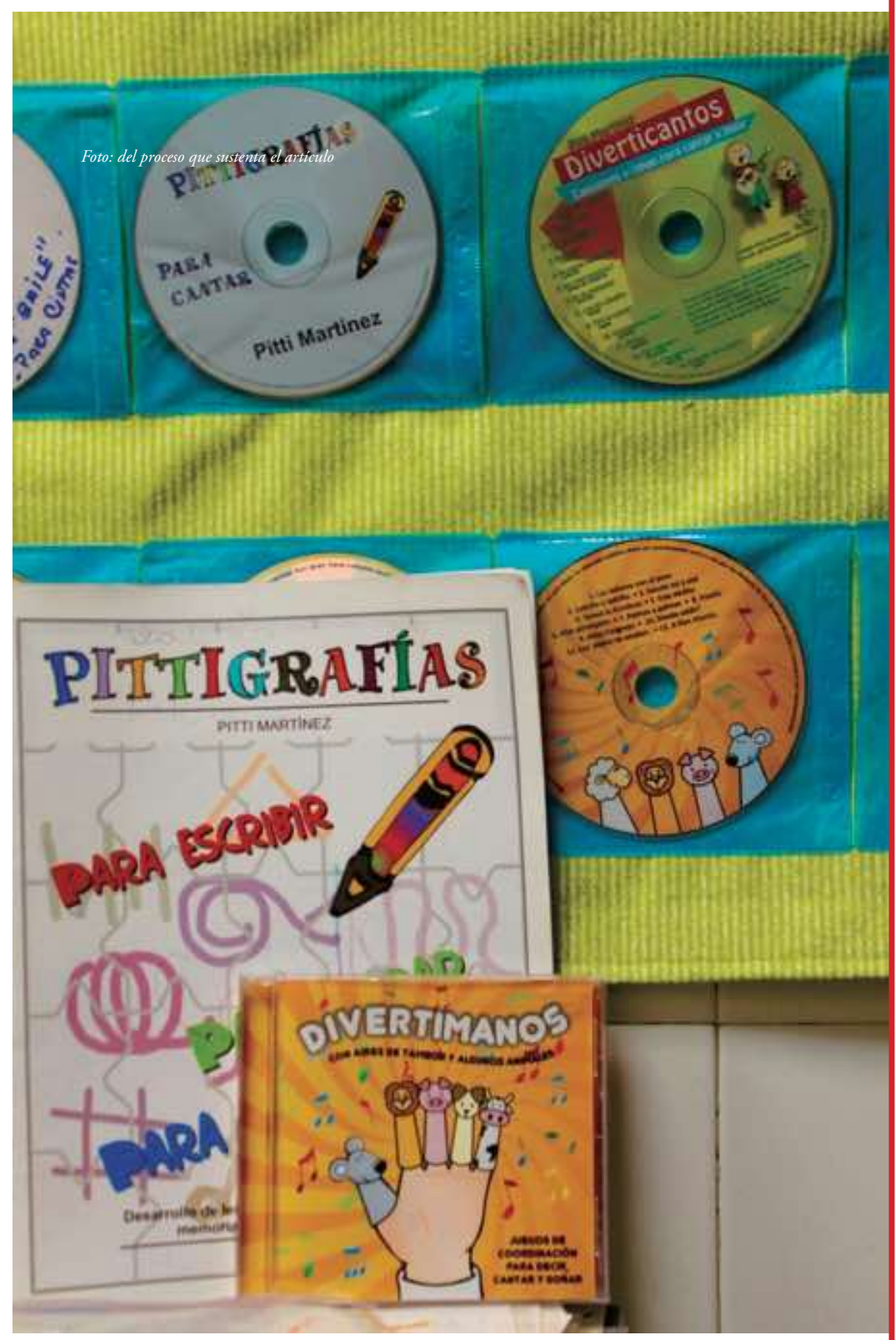

Aprestamiento desde la comprensión del hacer

El aprestamiento es una propuesta para la educación inicial desde la pedagogía musical que destaca como recursos la música, la tradición y la cultura. Busca que los niños estén preparados para la vida, porque brinda herramientas para fortalecer capacidades. Está estructurado en seis componentes dirigidos a favorecer el desarrollo lingüístico, motor, auditivo, musical, social y cultural. Implica un aprendizaje acumulativo y significativo, en un ambiente respetuoso, donde se generan entornos de oportunidad a través de retos posibles e instrucciones claras que propician orden en la secuencia de actividades y el disfrute de estas.

Durante el desarrollo de la experiencia se ha llevado a cabo un proceso de búsqueda, análisis, clasificación y selección de repertorios y materiales didácticos, parte de los cuales han sido compuestos y elaborados por la maestra y están condensados en varios $\mathrm{CD}$, cartillas y materiales ${ }^{8}$, todos ellos utilizados en las actividades de los diferentes niveles del aprestamiento. En la práctica ha sido posible identificar algunas constantes que reflejan no sólo intencionalidades pedagógicas, sino una estructura que ordena y secuencia los contenidos abordados de acuerdo a ejes del desarrollo musical y organiza las actividades y los repertorios, de acuerdo a edad y necesidades de estimulación en el área motriz, fonológica, auditiva, cognitiva y de relación social.

\section{Evolución de la estructura del aprestamiento}

Desde que inició la experiencia hasta la actualidad, los componentes del aprestamiento se han transformado en su concepción y ampliado en cuanto a sus posibilidades de acción para promover las capacidades de los niños. En principio, las clases se estructuraron en tres componentes: 1. El lenguaje: pensado en función del desarrollo de la articulación y la dicción de las palabras; 2. La audición: como ejercicio de discriminación tímbrica de juguetes y objetos sonoros, y 3. La ronda: para el desarrollo de actividades motrices básicas con canciones tradicionales. Posteriormente se dio una transformación del aprestamiento hasta llegar a la estructura actual que consta de seis componentes, como se puede observar en la tabla 1.

8 CD y Cartilla La magia de crecer (Martínez, 2001), selección de 16 canciones y actividades. Cartilla Pittigrafias. (Martínez, 2005) 42 ejercicios utilizados en el componente de pregrafía; CD Pittigrafias (Martínez, 2006), 42 rimas y canciones; CD Diverticantos (Martínez, 2008), 14 Canciones y rimas para cantar y jugar; Cartilla .pdf Diverticantos (Martínez, 2009), 14 ejercicios; CD Divertimanos.(Martínez, 2011), 12 canciones; CD y cartilla Pittigramas (Martínez, 2016), 17 rimas y canciones. 
Tabla 1

Evolución del aprestamiento

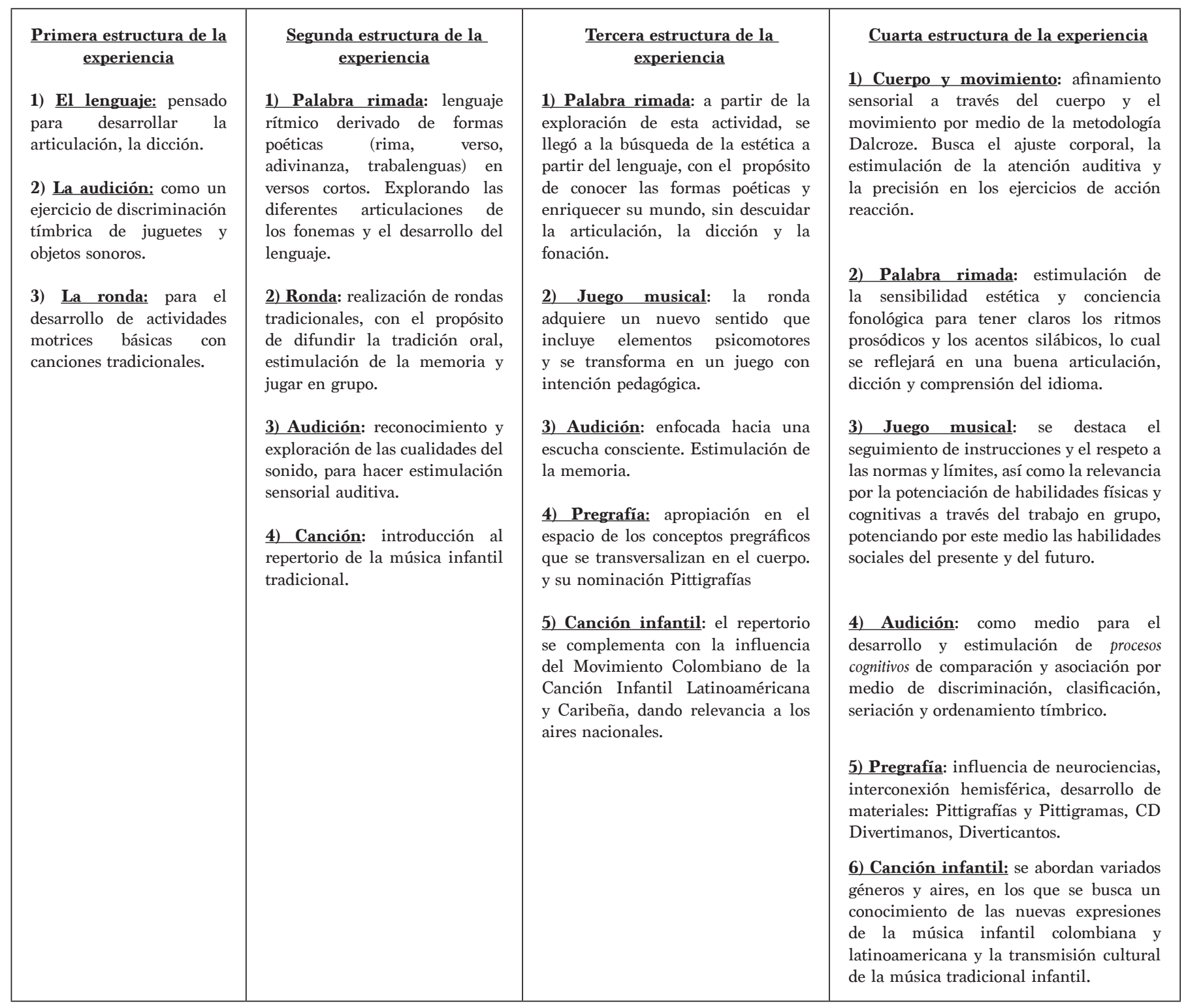




\begin{tabular}{|c|c|c|c|}
\hline $\begin{array}{l}\begin{array}{l}\text { Primera estructura de la } \\
\text { experiencia } \\
\text { Recursos y materiales }\end{array} \\
\text { 1) El lenguaje: rimas, versos, } \\
\text { adivinanzas, trabalenguas, } \\
\text { refranes. } \\
\text { 2) La audición: juguetes } \\
\text { sonoros e instrumentos de } \\
\text { pequeña percusión. } \\
\text { 3) La ronda: rondas } \\
\text { tradicionales. }\end{array}$ & $\begin{array}{l}\quad \begin{array}{l}\text { Segunda estructura de la } \\
\text { experiencia }\end{array} \\
\text { Recursos y materiales }\end{array}$ & $\begin{array}{l}\text { Tercera estructura de la experiencia } \\
\quad \text { Recursos y materiales } \\
\text { 1) Palabra rimada: rimas, versos, } \\
\text { adivinanzas, trabalenguas, refranes, } \\
\text { retahílas, jitanjáforas, aforismos, coplas, } \\
\text { aliteración, dichos, jeringonzas. } \\
\text { 2) Juego musical: rondas y juegos } \\
\text { musicales con el cuerpo como elemento } \\
\text { como elemento principal. Se incluyen aros, } \\
\text { pelotas de tamaño mediano, cintas, caballitos de } \\
\text { madera, títeres de dedos, narices de animales. } \\
\text { 3) Audición: objetos sonoros, } \\
\text { instrumentos de pequeña percusión, } \\
\text { grabaciones de sonidos de animales, de } \\
\text { la naturaleza y del entorno de su casa } \\
\text { y de la ciudad, animales de peluche con } \\
\text { sonido real, para poder realizar actividades de } \\
\text { discriminación y clasificación por el timbre y } \\
\text { biombo para la actividad de discriminación y } \\
\text { memoria auditiva. } \\
\text { 4) Pregrafía: palitos de madera, } \\
\text { plantillas y figuras de fomi, plantillas } \\
\text { de madera; pirámides, cubos y esferas } \\
\text { para ensartar; botones de colores para } \\
\text { ensartar y seguir secuencias. Tableros } \\
\text { personales y tizas. } \\
\text { Pittigrafias: cartilla con } 42 \text { ejercicios musicales } \\
\text { pregráficos y CD (composición y adaptación } \\
\text { Pitti Martinez). } \\
\text { 5) Canción infantil: compilación de } \\
\text { canciones infantiles tradicionales. } \\
\text { CD La Magia de crecer. } 16 \text { canciones infantiles } \\
\text { (Composición Pitti Martinez) } \\
\text { 14 juegosta etapa se emplea el CDciones (composición Pittti } \\
\text { Martinez) para acompañar las actividades de } \\
\text { los cinco componentes. }\end{array}$ & 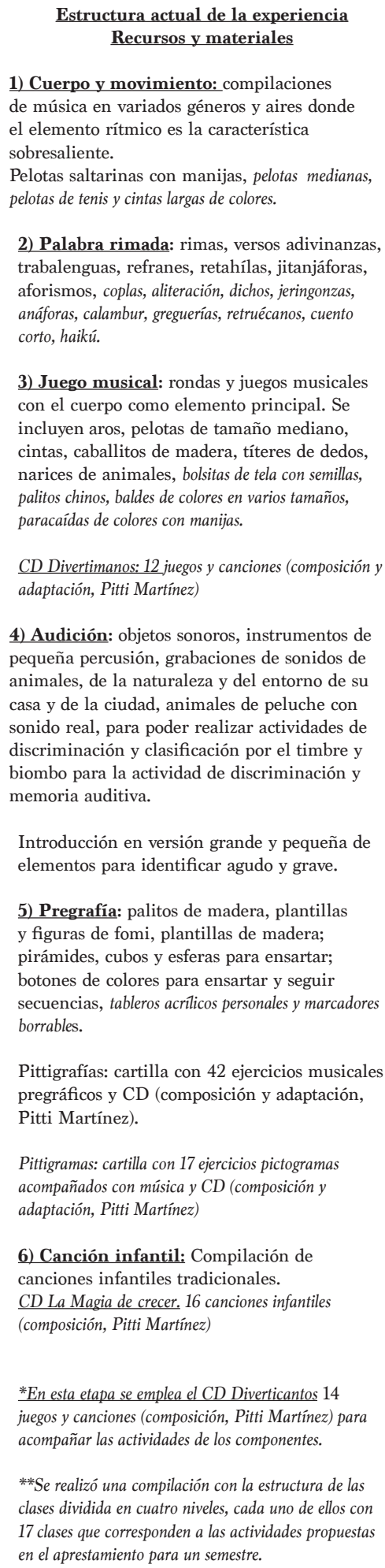 \\
\hline
\end{tabular}

Fuente: elaboración propia. 
Se observa en la tabla 1 que cada componente tiene un desarrollo implícito en el cual la maestra plasmó sus intencionalidades pedagógicas y musicales. También se evidencia que los componentes se han complementado y fortalecido durante la evolución del aprestamiento. A continuación se describen las características de los componentes.

\section{Componentes del aprestamiento}

La estructura actual de los seis componentes evidencia la reflexión sobre los saberes y prácticas en la experiencia, y a su vez pretende optimizar el empleo de los recursos, el manejo del tiempo y la pertinencia de las actividades y sus contenidos con el fin de favorecer el desarrollo de los niños.

Qué enseñar y qué aprender de forma significativa son cuestionamientos permanentes que guían la selección de repertorios de las actividades en los componentes. Se requiere, por tanto, encontrar el equilibrio entre las necesidades de los niños, en cuanto a su formación específica musical y las características de su desarrollo, que les permita relacionarse con ellos mismos y con su entorno, y mejorar su capacidad cognitiva sin perder el sentido del goce. El aprestamiento se convierte en una forma novedosa y creativa de enseñar a aprender, con el valor agregado de que la música empieza a ser parte de sus vidas.

1. Componente cuerpo y movimiento (sensibilización al mundo sonoro y expresivo) Se plantea al inicio de la clase para permitir a los niños estar dispuestos física y emocionalmente en las actividades. Con el movimiento se liberan tensiones físicas y emocionales y se generan situaciones de bienestar y alegría. Se propicia la relación cuerpo y música, vinculándola a la audición, usando diversos géneros, con un repertorio de canciones previamente seleccionadas. Se trabaja específicamente en la adquisición de equilibrio, agarre y precisión visomotora, direccionalidad y coordinación gruesa, a través del seguimiento de instrucciones, las cuales los niños deben escuchar y decodificar. Este componente es guiado por elementos de la metodología Dalcroze (Pascual, 2002; Veltri, 1969; Rodrigues, 2011).

\section{Componente palabra rimada (comunicación asociativa y rítmica)}

Contiene actividades de recitado rítmico en las que se emplea el lenguaje para acercar el niño a la música y por este medio mejorar su emisión vocal. Promueve la atención, concentración y memoria implicada en el aprendizaje de las palabras y los agrupamientos rítmicos de estas. En este apartado se evidencia la búsqueda de posibilidades estéticas venidas del lenguaje y la asociación de este con el ritmo, por medio de los cuales se incentiva en los niños la imaginación, el asombro y la fantasía. Esta es una forma de aproximarse a manifestaciones artísticas que permiten aprender el lenguaje, apropiarse de su mensaje e introducirse en la experiencia estética al ser partícipes de esta. Este componente es guiado por elementos de la metodología Orff (Riaño, Díaz, Ibarretxe, García, Malbran, 2011; Pascual, 2002; Orff, 1950).

\section{Componente juego musical (interacción lúdica)}

La intencionalidad pedagógica presente en el juego musical se evidencia en el desarrollo de contenidos psicomotrices, por medio de los cuales se promueve el conocimiento del cuerpo y su relación con el espacio, todo esto mediado por la música, sus cualidades y el gusto que se genera a través de la escucha (Escudero, 1988).

En las actividades de este componente se da una toma de conciencia sobre la responsabilidad social en la preservación de la cultura, valores, imaginarios de identidad nacional, en los que se toma al juego musical como una herramienta para tal fin. A través del juego se plantean escenarios de oportunidad en los que se pueden vivenciar expresiones artísticas y culturales, generar lazos de significatividad e identidad, además de brindar sentido y color a la vida de los niños. Este componente es guiado por aportes de Vahos (1998, 2000), Marulanda (1988), Posada (2000), y Wirth, Stassevitch, Shotwell y Stemmler (1983).

\section{Componente audición (relación audiosonora)}

Las actividades de este componente se enfocan hacia una escucha conciente, mediante un proceso gradual, con ejercicios de estimulación sensorial (oír) y de dirección de atención auditiva (escuchar), para incitar al desarrollo del pensamiento asociativo, secuencial, comparativo, lógico y musical, promoviendo también la memoria, la atención y la concentración (Aguirre, 2005).

Se estimula la percepción auditiva fina de los niños, es decir, la capacidad de percibir las cualidades del sonido, su densidad, establecer relaciones entre ellas y aumentar la capacidad asociativa que permite recordar más clara y eficientemente lo escuchado. Pone de manifiesto la importancia que se le ha dado al desarrollo auditivo, que incide directamente en los procesos neurológicos asociados a la escucha consciente, la cual contribuye a sentar las bases para aprender a aprender, de forma eficiente y eficaz.

La escucha consciente en el aprestamiento se da a través de la implementación de elementos de apoyo (objetos sonoros e instrumentos de pequeña percusión), creando un puente asociativo entre imagen, nombre y timbre, constituyéndose en una experiencia significativa, con la cual se podrá hacer transferencias a nuevos conocimientos (Willems, 2001).

Los sentidos e intencionalidades de este componente se ven explicitados, en palabras de la maestra, así:

Este es un momento de magia para los niños, la actividad aproximadamente es de 10 minutos pero en realidad les va a durar para toda la vida, porque en este momento es cuando ellos descubren y aprenden cómo dirigir su atención auditiva y cómo desarrollar pensamiento asociativo a partir de la audición. Esto requiere un mayor esfuerzo de su parte, el trabajo auditivo, que empieza a los dos años con los juguetes de plástico como vaca, pato, perro etc., pasa a peluches de animales con sonidos reales y se va complejizando hasta llegar a objetos sonoros y luego a instrumentos musicales. El proceso se plantea según las necesidades de cada niño (entrevista a la maestra Pitti Martínez).

Este componente es guiado por la metodología Willems (1981, 2001) y aportes del Método Tomatis (2016) .

\section{Componente pregrafia ${ }^{10}$ (conceptos y asociaciones psicomotoras)}

9 Se puede consultar información sobre este método en el sitio web http://www.tomatis.com/ 10 En el Aprestamiento el componente pregrafía, hace referencia a la preparación de la escritura y la lectura del lenguaje de la música, más conocido como solfeo, consistente en el sistema de notación para las cualidades del sonido, como lo son altura (las variaciones de altura constituyen a las melodías), timbre (cualidad que permite identificar las sonoridades de las vibraciones), intensidad (referente a las variaciones dinámicas) y duración (que tiene que ver con las variaciones de durón e

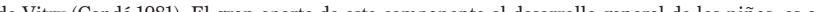
de Vitry (Cande, 1981). El gran aporte de este componente al dos el empleo del cuerpo como medio de apropiación y asociación de los conceptos psicomotores previos
a la escritura (Escudero, 1988), con el soporte, estímulo y dinamización de la música. Este ejercicio se constituye en una transposición didáctica en los procesos escolares que presentan similitudes tales como: lectura, escritura, matemáticas, geometría, español y literatura, entre otras. 

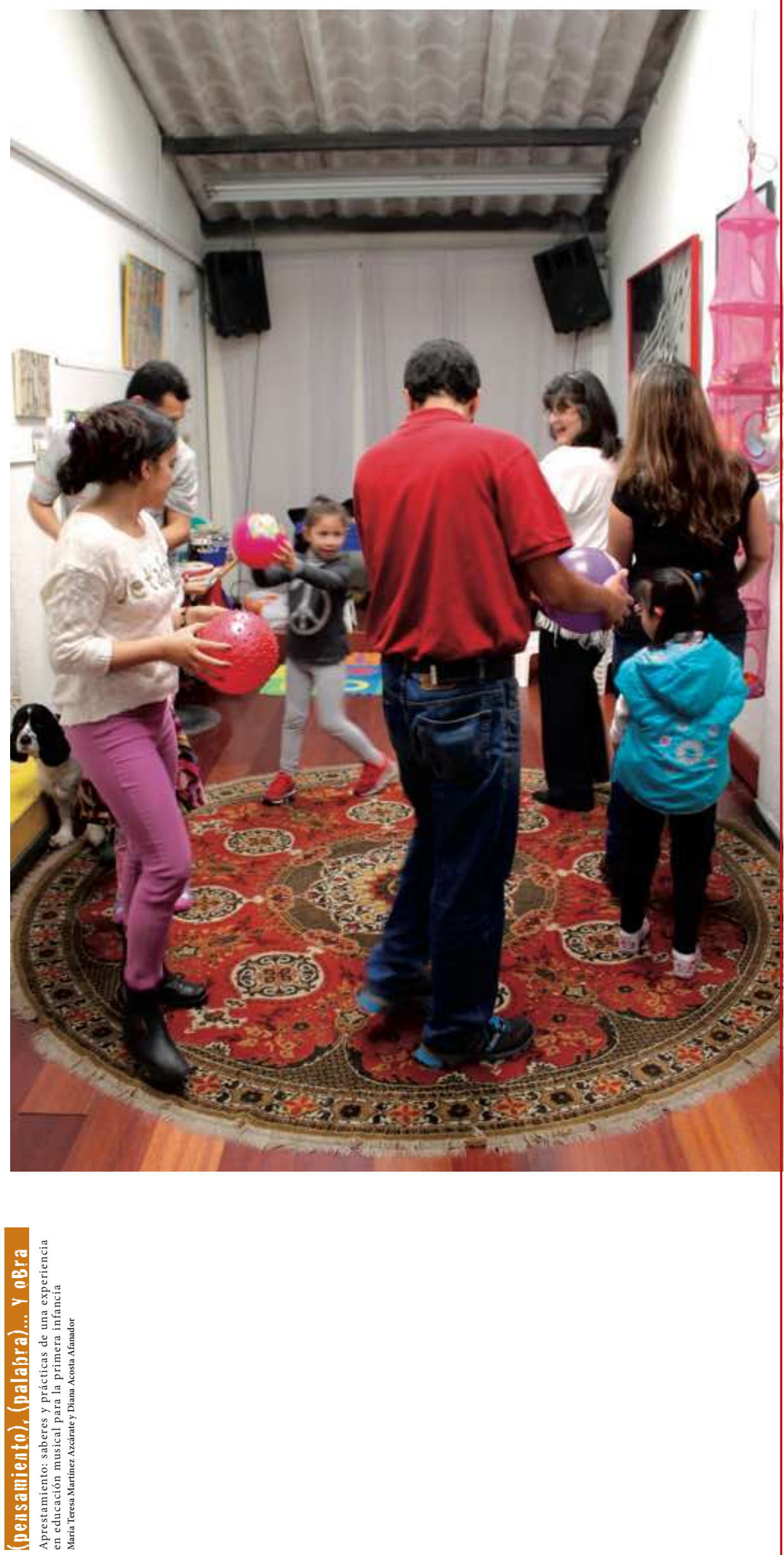

Implica la interiorización de conceptos previos a la escritura, vivenciados en el cuerpo. Las actividades son acompañadas con música y movimiento expresivo, gracias a lo cual se genera un entorno de oportunidad para la exploración de grafías como una consecuencia y síntesis de los demás componentes del aprestamiento.

Cantar y graficar refleja la conexión de la música y los elementos percibidos. Es una actividad constante para crear asociaciones entre la escucha consciente y la motricidad fina. Se emplea el cuerpo, el lenguaje, la canción y el movimiento, en una sucesión de actividades que conforman la ruta pregráfica del aprestamiento:

Escuchar: se centra en la emoción que produce el sonido, es lo que se siente al percibirlo sin más implicaciones. Es una vivencia de los elementos que constituyen la música que acompaña el proceso pregráfico. Es una escucha no condicionada y libre.

Asociar movimiento: expresar con el cuerpo los elementos musicales percibidos empleando conceptos de espacialidad y direccionalidad. Requiere del seguimiento de instrucciones.

Cantar y asociar: se asocia el movimiento a la direccionalidad del trazo pintado en el aire empleando primero una mano, luego la otra y finalmente ambas. En este paso siempre está presente el lenguaje hablado o cantado. Requiere el aprendizaje del texto que acompaña la música.

Cantar y graficar: refleja la conexión de la música y los elementos percibidos

El recurso principal para las actividades de este componente es la cartilla y CD diseñado por la maestra denominado Pittigrafías (Martínez, 2005, 2006) y los Pittigramas (Martínez, 2016). Este componente cuenta como referentes a Ercilia de Calvo (1981) y Tita Maya (2001).

\section{Componente canción infantil (vínculo afectivo)}

Este componente toma a la imitación como recurso y promueve la entonación, la respiración consciente, la articulación y comprensión de todas las palabras cantadas e invita a dirigir la atención hacia la línea melódica, la expresión y la aproximación al contorno sonoro de la canción, que es el comienzo de la afinación en los niños, además fortalece elementos para que los niños se acerquen a la tradición y cultura (Pascual, 2002 pp. 122-149). Se destaca en este componente la importancia de contar con un repertorio dirigido y adecuado para los niños, que estimule el lenguaje, la fantasía, su expresión, el desarrollo vocal y la alegría (Martenot, 1993, p. 31). 
En el aprestamiento la canción es un elemento cohesionante para la familia, al generar lugares comunes entre ellos y el niño, porque se dan oportunidades para compartir y comunicarse usando la canción infantil como pretexto.

Para la selección del repertorio, se han considerado los siguientes criterios:

a) Emplear textos cortos, preferiblemente con fonemas repetidos.

b) Emplear onomatopeyas como recurso para mejorar la articulación del lenguaje. c) Escoger canciones que contengan elementos del ritmo (pulso, acento y división), de las cualidades del sonido (altura, timbre, intensidad y duración), motrices, de esquema corporal, y que sean susceptibles de expresarse corporalmente con mímica.

d) Escoger canciones tradicionales y que promuevan aires nacionales.

La canción está presente como recurso en las metodologías musicales del siglo $\mathrm{xx}$ (Pascual, 2002, pp. 239-256), además estimula el desarrollo del lenguaje y la comprensión del mismo, a través de las formas literarias contenidas en las canciones y la conciencia fonológica desprendida de la apropiación de los textos en ellas contenidos. Está presente también la estimulación de la imaginación y la fantasía, por medio de las cuales se generan relaciones sustantivas entre lo que se conoce y lo que se aprende, y se van estructurando rutas asociativas venidas del asombro y la alegría de cantar.

La canción infantil, además de contener valor lúdico y de preservación de la tradición y la cultura (Corral, 2009), fortalece aspectos emocionales y sociales porque permite que los niños se conecten con los demás y desarrollen empatía. Este componente es guiado por las metodologías Willems y Kodaly (Zuleta, 2003, 2008, 2009, 2014).

El aprestamiento en clave de sus aportes

A lo largo del proceso de la sistematización del aprestamiento, se determinó que esta es una experiencia de características únicas, por la confluencia de factores pedagógicos, personales y profesionales de la maestra, quien genera a partir de entornos de oportunidades sugerencias de uso, adaptación y creación de variados recursos musicales y pedagógicos, en los que cualquier agente educativo puede inspirarse para ser el mediador entre las necesidades de los niños y sus característica de desarrollo, según las posibilidades que le ofrece el contexto.
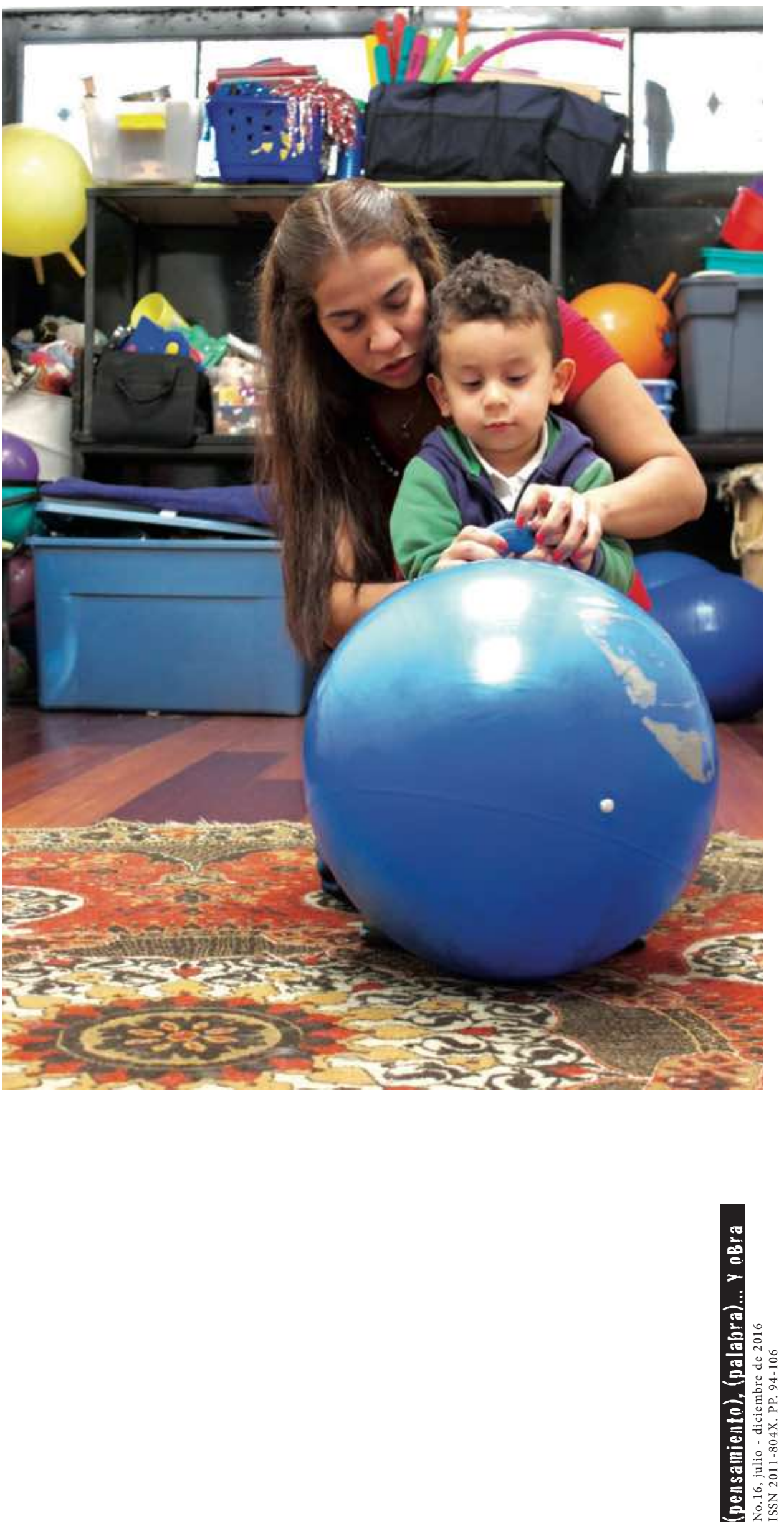
Se observó que las actividades en el aprestamiento se desenvuelven a través de hilos conductores, aparentemente invisibles, pero manifiestos en forma de secuencias que dan sentido y orden a las acciones dentro de cada uno de los seis componentes, para hacer más asertiva la planeación y la selección de actividades y establecer también por su mediación una suerte de rutas metodológicas para el desarrollo de la clase.

Estas secuencias se presentan en tres momentos que contribuyen a la comprensión del hacer y son una sumatoria de saberes representados con palabras que identifican y caracterizan las acciones y propósitos subyacentes, dirigidos al maestro, al niño y al proceso pedagógico en desarrollo.

En la tabla 2 se presentan algunas secuencias que ilustran las intencionalidades pedagógicas subyacentes en las prácticas del aprestamiento:

Tabla 2

Intencionalidades pedagógicas subyacentes en las prácticas del aprestamiento

\begin{tabular}{|c|c|c|}
\hline Momento 1 & Momento 2 & Momento 3 \\
\hline $\begin{array}{c}\text { Imitar } \\
\text { Lo que ve y oye }\end{array}$ & $\begin{array}{c}\text { Comprender } \\
\text { El movimiento, el sonido, la forma, la } \\
\text { secuencia, por los sentidos, la kinestesia y la } \\
\text { propiocepción }{ }^{1}\end{array}$ & $\begin{array}{l}\text { Integrar } \\
\text { Conceptos, nombres, relaciones, } \\
\text { cualidades }\end{array}$ \\
\hline $\begin{array}{c}\text { Oír } \\
\text { Acto fisiológico }\end{array}$ & $\begin{array}{l}\text { Escuchar } \\
\text { Atención hacia el fenómeno sonoro, como } \\
\text { proceso voluntario-activo. }\end{array}$ & $\begin{array}{l}\text { Asociar/memorizar } \\
\text { Nombre, imagen, timbre, secuencia }\end{array}$ \\
\hline $\begin{array}{c}\text { Yo } \\
\text { Reconocimiento de las propias } \\
\text { posibilidades y limitaciones }\end{array}$ & $\begin{array}{c}\text { El otro } \\
\text { Respeto, interacción, encuentro }\end{array}$ & $\begin{array}{c}\text { Los otros } \\
\text { Trabajo de equipo, responsabilidad, } \\
\text { comunidad }\end{array}$ \\
\hline $\begin{array}{c}\text { Disciplina } \\
\text { Normas y límites }\end{array}$ & $\begin{array}{c}\text { Constancia } \\
\text { Afinamiento motor, auditivo, vocal }\end{array}$ & $\begin{array}{c}\text { Paciencia } \\
\text { Retos posibles, logros para todos }\end{array}$ \\
\hline $\begin{array}{c}\text { Emocional } \\
\text { Lúdica, resiliencia, } \\
\text { sensibilidad, empatía, afecto }\end{array}$ & $\begin{array}{c}\text { Cognitivo } \\
\text { Convergencia, divergencia, interconexión } \\
\text { hemisférica }\end{array}$ & $\begin{array}{c}\text { Social } \\
\text { Ciudadanía, tradición, cultura, respeto }\end{array}$ \\
\hline $\begin{array}{c}\text { Sistemático } \\
\text { Orden, estructura, }\end{array}$ & $\begin{array}{c}\text { Integral } \\
\text { Desarrollos }\end{array}$ & $\begin{array}{l}\text { Participativo } \\
\text { Para todos }\end{array}$ \\
\hline $\begin{array}{l}\text { Planear } \\
\text { Selección de repertorios y } \\
\text { recursos }\end{array}$ & $\begin{array}{l}\text { Hacer } \\
\text { Didáctica }\end{array}$ & $\begin{array}{c}\text { Verificar } \\
\text { Reflexión, comparación }\end{array}$ \\
\hline $\begin{array}{l}\text { Eficacia } \\
\text { En el hacer }\end{array}$ & $\begin{array}{c}\text { Eficiencia } \\
\text { En el empleo de los recursos }\end{array}$ & $\begin{array}{c}\text { Pertinencia } \\
\text { En los contenidos }\end{array}$ \\
\hline $\begin{array}{c}\text { Sentir } \\
\text { Cualidades, formas }\end{array}$ & $\begin{array}{c}\text { Hacer } \\
\text { El cuerpo, la voz, elementos }\end{array}$ & $\begin{array}{c}\text { Memoria } \\
\text { Muscular, lingüística, musical }\end{array}$ \\
\hline
\end{tabular}

Fuente: elaboración propia.

1 Riaño, Díaz, Ibarretxe, García y Malbrán (2011) afirman que las propiocepciones brindan información sobre la postura del cuerpo y "permiten hacernos consciente de nosotros mismos y de nuestros movimientos. En última instancia, nos sirven para regular y conceptualizar experiencias corporales como la tensión o la relajación de nuestros músculos, la fuerza, el equilibrio, la presión, la posición, etc. Por ello la conceptualización y la comprensión de los fenómenos musicales también estará relacionada con respuestas fisiológicas determinadas". (p. 53)

Queda abierta la invitación para que cada agente educativo encuentre sus propias secuencias, y dé mayor claridad y comprensión a su hacer.

\section{Sentidos y comprensiones emergentes}

Se encontró que en el aprestamiento existe coherencia pedagógica entre el discurso que sustenta el hacer, la práctica misma y la reflexión realizada sobre esta. Se evidencia la importancia dada en la experiencia, no sólo a la construcción del conocimiento, sino al contexto, a la tradición y la cultura, todo esto mediado por el lenguaje, en un ambiente natural y afectivo, reconociendo en cada niño sus diferencias individuales, sus necesidades evolutivas y sus potencialidades en lo musical, vinculando además en este proceso a la familia.

Tener un acercamiento al aprestamiento desde una perspectiva investigativa permitió tomar consciencia de su estructura en seis componentes, de los saberes que guían las prácticas y de las secuencias que sustentan el hacer. Se hallaron además otros elementos valiosos en la experiencia, como lo son: 
1. Hacer énfasis en vivir y sentir, como punto de partida en las actividades.

2. Sensibilizar a los niños, a través del cuerpo y el movimiento, al mundo sonoro y expresivo que los rodea.

3. Establecer formas de interacción lúdica con la música y aprender que hay otras maneras de relacionarnos con los otros. 4. Promover la escucha consciente que lleve al disfrute de la música y a hacerla parte de la vida.

5. Apropiar la tradición y la cultura del país a través de la canción infantil en aires colombianos.

6. Invitar al maestro a tomar consciencia de que es un artista en el momento de hacer música y que es arte lo que debe proyectar.

7. Lograr que los niños sientan el deseo de permanecer y volver a clase.

8. Considerar a la familia como elemento integrador de la experiencia musical y los saberes recibidos en clase.

9. Aprender formas sencillas de jugar, maneras simples de hallar felicidad y alegría a través de la música y el arte.

$\mathrm{El}$ aprestamiento pone a los niños en contacto con el mundo sonoro y contribuye al desarrollo de la percepción del entorno. Como consecuencia, se logra la apropiación del yo a través del sentir, del hacer, del aprender a hacer bien, de aceptar y valorar, de reconocer limites propios y ajenos, y por este medio, descubrir una parte del mundo y a sí mismo. En la realización de cada componente se hace presente la imaginación y la fantasía, promovida por los textos de las canciones, el asombro, el descubrimiento del mundo a partir del trabajo integrado con los sentidos, a través del momento lúdico y creativo en la interacción con el medio, los otros, y por último, la generación de vínculos entre sus pares y acompañantes.

La música entonces es una herramienta que cobra relevancia en cualquier proceso de formación, porque permite diversos usos para el desarrollo de actividades desde cualquier área del conocimiento.

Entonces en el aprestamiento se reconoce al niño como un campo de potencialidad para ser, hacer y conocer, al hacerlo partícipe de experiencias de calidad y que propicien la alegría de la vida y el desarrollo humano. Así se ven estimuladas sus capacidades cognitivas, físicas, sociales, artísticas, musicales y emocionales, lo cual repercute positivamente en sus vidas.

Existe entonces la necesidad de cualificar el desempeño del maestro, en términos de seleccionar de forma adecuada y eficiente los contenidos de acuerdo a los objetivos propuestos. También para ser eficaz en el empleo de los recursos y la pertinencia de las actividades. Por tanto, el aprestamiento invita a que estas se dirijan hacia:

1. El goce lúdico y la alegría de la vida.

2. Promover sentido estético por el encuentro con modelos de calidad.

3. Transmisión y preservación cultural.

4. Promoción de normas y valores.

5. Respeto y reconocimiento de sí.

6. Respeto y reconocimiento del otro.

7. Identificación y reconocimiento de las particularidades y características de los niños.

8. Generación de retos posibles y afianzamiento de la autoestima.

9. Crear entornos de oportunidad para optimizar el hacer y los escenarios de aprendizaje, favoreciendo en el niño: a. El encuentro de la familia alrededor de la música

b. Los vínculos afectivos consigo mismo, con los demás y con la música

c. El desarrollo del pensamiento

d. La estimulación de la memoria

e. La atención sostenida en el presente.

f. La disposición para enfrentar retos futuros en la vida académica y social

g. La detección de posibles talentos para la música

Se encuentra que la sistematización de la experiencia del aprestamiento, se constituye en un ejercicio reflexivo que invita al maestro:

A que evite pensar su labor en términos de actividad lúdica o recreativa únicamente, y se empodere como artesano de sueños y cultura, que moldea pensamientos y emociones.

- A poner el conocimiento al alcance de los niños, de una forma cercana y sencilla.

A propiciar el desarrollo de las capacidades de los niños por medio de lenguajes artísticos y la interiorización de la tradición, la cultura, valores y normas.

A ser coherente y fiel a lo que se es, a los propios sentimientos, contextos, deseos, conocimientos, a su propia historicidad, para hacer que sus prácticas se conviertan en una expresión de su autenticidad.

- A realizar una autorreflexión constante sobre los contenidos, recursos y decisiones de sus prácticas.

A cualificarse con saberes aportantes a sus prácticas. A incluir nuevos conocimientos y disciplinas que enriquezcan su hacer.

- A lograr que la música forme parte de la vida de los niños.

A crear materiales didácticos (impresos, grabaciones, audiovisuales y otros objetos de apoyo) acordes con sus intencionalidades pedagógicas y su saber.

A traer la magia y la alegría a los niños y a sus propias vidas, para que sin importar las complejidades de sus contextos, puedan hallarle sentido a la vida y dar esperanza.

- A crear experiencias como alternativas de transformación de personas y comunidades, para contribuir al presente y tener diferentes perspectivas en el futuro.

Para finalizar, debemos tener presente el compromiso social de los maestros en la formación de seres humanos sensibles y empáticos que puedan reconocerse a sí mismos y a los demás con sus diferencias, fortalezas y debilidades, para lo cual es fundamental propiciar que la música y el arte hagan parte de sus vidas. 
- Acosta, D. y Martínez, M. (2016). Saberes y prácticas de una experiencia musical para la primera infancia en la ciudad de Bogotá. (Monografía de Maestría). Universidad Pedagógica Nacional. Bogotá.

- Aguirre, I. (2005). Teorías y prácticas en educación artística. España: Limpergraf.s.l.

- Calvo, E. (1981). La música y el ritmo en el mundo del principito. Cali: Edicol.

- Candé, R. (1981). Historia Universal de la Música. Madrid: Aguilar.

- Corral, M. (2009). Arte y expresión en los primeros años. Grandes temas para los más pequeños, acerca de la complejidad en la educación en los primeros años. Buenos Aires: Puerto Creativo.

- Escudero, M. (1988). Educación musical, rítmica y psicomotriz. Madrid: Real Musical.

- Ghiso, A. (1999). De la Practica singular al dialogo con lo plural. Aproximaciones a otros tránsitos y sentidos de la sistematización en épocas de globalización. La Piragua. Revista Latinoamericana de Educación, (16), 5-12. Recuperado de http://www.ceaal.org/v2/ archivos/publicaciones/piragua/Docto14.pdf

- Mannes, E. (2009). The Music Instinct: Science and Song. Recuperado de https://www.youtube.com/watch?v=m5pwSMDTD4M

- Martenot, M (1993). Principios fundamentales de formación musical y su aplicación. Madrid: Rialp.

- Martínez, P. (2001). La magia de crecer Canciones para niños con corazón grande. (CD audio). Bogotá: Play Record.

- Martínez, P. (2003). La magia de crecer. Bogotá: Representaciones HRS.

- Martínez, P. (2005). PITTIGRAFÍAS PARA ESCRIBIR PARA JUGAR PARA CANTAR Desarrollo del lenguaje-motricidad finaatención-memoria-preparación-a la escritura. Bogotá: Giro Editores.

- Martínez, P. (2006). Pittigrafías Canciones y rimas para cantar-oír -escribir-jugar-leer-reír y soñar. Música para todos. (CD audio). Bogotá: IN TONE.

- Martínez, P. (2006). Pittigrafías Canciones y rimas para cantar-oír -escribir-jugar-leer-reír y soñar. Música para todos. (DVD). Bogotá: IN TONE.

- Martínez, P. (2008) Diverticantos Canciones y rimas para cantar y jugar. (CD audio). Bogotá: IN TONE.

- Martínez, P. (2009). Diverticartilla Cartilla complementaria del CD Diverticantos Canciones y rimas para cantar y jugar. Bogotá.

- Martínez, P. (2011). Divertimanos Con aires de tambor y algunos animales Juegos de coordinación para decir, cantar y soñar. (CD audio). Bogotá: IN TONE.

- Martínez, P. (2016). Pittigramas Pictogramas para cantar y decir con guitarra y algo más. (CD Audio y cartilla). Bogotá: IN TONE.

- Marulanda, O. (1988). Las rondas y los juegos infantiles folclor y educación. Bogotá: Gente Nueva.

- Maya, T. (2001). Garabateo de la gramática musical. Medellín: Corporación Cantoalegre.

- Orff, C. (1950). Música para niños. Obra didáctica de Carl Orff. Buenos Aires: Ricordi.

- Pascual, P. (2002). Didáctica de la música para primaria. España: Prentice Hall.

- Posada, P. (2000). Cantar, tocar y jugar Juegos musicales para niños. Medellín: Editorial Universidad de Antioquia.

- $\quad$ Riaño, M., Díaz, M., Ibarretxe, G., García, E., Malbran, S. (2011) Fundamentos musicales y didácticos en educación infantil. España: Ediciones de la Universidad de Cantabria.

- $\quad$ Rodrigues, I. (2011). Memorias del Diplomado de la Universidad Pedagógica Nacional La Rítmica Dalcroze de hoy-Una educación por y para la música. Bogotá: Universidad Pedagógica Nacional.

- Torres, A. (1998). La sistematización de experiencias educativas reflexiones de una práctica reciente. Ponencia presentada para el Tercer Congreso Iberoamericano y de Agentes de Desarrollo Sociocultural y Comunitario. La Habana, Cuba. Recuperado de http://www.pedagogica.edu.co/ storage/ps/articulos/pedysab13_04arti.pdf

- Vahos, O. (1998). Juguemos - 71 juegos infantiles predancísticos, preteatrales, musicales, teoría del juego, retahilas, trabalenguas, adivinanzas, conteos, partituras. Medellín: Real Cultura.
- Vahos, O. (2000). Juguemos Dos- Juego y pedagogía, Cerebroendorfinas-lúdica-arte, 45 Juegos infantiles de Colombia, lúdica verbal, origami. Medellín: Real Cultura.

- Veltri, A. (1969). Apuntes de didáctica. Buenos Aires: Editorial DAIAM.

- Willems, E. (1981). El valor humano de la educación musical. Barcelona: Paidós.

- Willems, E. (2001). El oído musical: la preparación auditiva del niño. Barcelona: Paidos Iberica.

- Wirth, M., Stassevitch, V., Shotwell, R., y Stemmler, P. (1983). Musical games, fingerplays and rhythmic activities for early childhood. New York: Parker Publishing Company, Inc.

- Zuleta, A. (2008). El método Kodaly en Colombia. Bogotá: Editorial Pontificia Universidad Javeriana.

- Zuleta, A. (2009). Antología Kodaly Colombiana. Bogotá: Editorial Pontificia Universidad Javeriana.

- Zuleta, A. (2014). Antología Kodaly Colombiana II. Bogotá: Editorial Pontificia Universidad Javeriana.

\section{Pitti Martínez (María Teresa Martínez Azcárate)}

Licenciada en Pedagogía Musical de la Universidad Pedagógica Nacional. Compositora, intérprete, directora coral, docente de la Licenciatura en Música de la Universidad Pedagógica Nacional. Miembro de la Junta Directiva del Foro Latinoamericano de Educación Musical (FLADEM), Colombia, y miembro del grupo de investigación Corpus Teórico de la Facultad de Bellas Artes de la Universidad Pedagógica Nacional. Candidata a magíster en Desarrollo Educativo y Social en el convenio entre el Centro Internacional de Educación y Desarrollo Humano y la Universidad Pedagógica Nacional. Correo electrónico: pittimusica@gmail.com

\section{Diana Acosta Afanador}

Abogada de la Universidad Nacional, funcionaria de la Oficina Jurídica de la Universidad Pedagógica Nacional y candidata a magíster en Desarrollo Educativo y Social en el convenio entre el Centro Internacional de Educación y Desarrollo Humano y la Universidad Pedagógica Nacional. Correo electrónico: 323daa@gmail.com

Artículo recibido en enero de 2016 y aceptado en marzo de 2016 